\title{
Therapeutic Potential of Curcumin for the Treatment of Malignant Gliomas
}

\author{
Blake C. Walker ${ }^{1,2} \bullet$ Srijan Adhikari ${ }^{1,2} \bullet$ Sandeep Mittal ${ }^{1,2}$ \\ ${ }^{1}$ Fralin Biomedical Research Institute, Virginia Tech Carilion School of Medicine, Roanoke, \\ VA, USA; ${ }^{2}$ Carilion Clinic - Neurosurgery, Roanoke, VA, USA
}

Author for correspondence: Sandeep Mittal, Virginia Tech Carilion School of Medicine, 2331 Franklin Rd SW, Suite 2154, Roanoke, VA, USA. Email: sandeepmittal@vt.edu

Doi: https://doi.org/10.36255/exonpublications.gliomas.2021.chapter8

\begin{abstract}
Glioblastoma is the most common primary malignancy of the central nervous system. Maximal surgical resection of glioblastoma in addition to temozolomide and fractionated radiation therapy provides an overall median survival of approximately 15 months. The addition of tumor-treating fields (Optune therapy) has the potential to increase median survival to 20 months, although compliance and ease of use remain an issue. Glioblastoma remains a devastating diagnosis fraught with complications. Curcumin is a yellow pigment from the rhizome of the ubiquitous and commercially available spice, turmeric (Curcuma longa). Turmeric has been long used in Indian traditional medicines and has been established as a safe food additive by the US Food and Drug Administration. There is a wealth of in vitro data suggesting that turmeric's main active component, curcumin, has many favorable effects on glioblastoma. Curcumin has been shown to potentiate the effects of chemotherapy and radiation, decrease malignant spread, protect normal tissue from oxidative stress, and regulate many genetic targets resulting in glioblastoma cell death. Curcumin's positive safety profile and potential therapeutic effects on glioblastoma make it a promising potential adjunct to current standard treatment regimens.
\end{abstract}

Keywords: anti-glioma activity; central nervous system tumors; curcumin; glioblastoma; high-grade gliomas

In: Gliomas. Debinski W (Editor). Exon Publications, Brisbane, Australia. ISBN: 978-0-6450017-4-7; Doi: https://doi.org/10.36255/exonpublications.gliomas.2021

Copyright: The Authors.

License: This open access article is licenced under Creative Commons Attribution-NonCommercial 4.0 International (CC BY-NC 4.0) https://creativecommons.org/licenses/by-nc/4.0/ 


\section{INTRODUCTION}

Gliomas have historically been divided into 4 grades by WHO classification, with highly variable prognosis between the histologic grades. WHO grade I gliomas include pilocytic astrocytoma, subependymoma, subependymal giant cell astrocytoma, ganglioglioma, desmoplastic infantile astrocytoma, and myxopapillary ependymoma, among others. WHO grade II gliomas are infiltrative and generally more aggressive than grade I tumors and include oligodendroglioma, fibrillary astrocytoma, pleomorphic xanthoastrocytoma, and mixed oligoastrocytoma, as well as other less common tumor types. WHO grade III gliomas include anaplastic astrocytoma, anaplastic oligoastrocytoma, anaplastic ganglioglioma, anaplastic ependymoma, and anaplastic pleomorphic xanthoastrocytoma. Finally, WHO grade IV gliomas include glioblastoma, various subtypes of glioblastoma, and pinealoblastoma (1). Within the category of diffuse, infiltrating gliomas (WHO II, III, IV), tissue diagnosis was traditionally made based on histopathologic analysis based on the presence or absence of microvascular proliferation, necrosis, and mitotic activity. The introduction of genetic profiling of gliomas based on IDH mutation, MGMT promoter methylation status, $1 \mathrm{p} / 19 \mathrm{q}$ codeletion has led to better understanding and more appropriate classification of gliomas and their subtypes (1).

With an annual incidence of 3.21 per 100,000 individuals, glioblastomas are the most common primary malignant brain tumor with nearly 11,000 new cases diagnosed each year in the United States (2). According to the CBTRUS data repository, glioblastoma accounted for $14.5 \%$ of all CNS tumors, $57.7 \%$ of all gliomas, and 48.6\% of all malignant CNS tumors diagnosed from 2013-2017 (2). Prognosis for patients with glioblastoma remains dismal. Aggressive maximal resection followed by radiotherapy has a median overall survival of 12.1 months. The addition of adjuvant alkylating chemotherapy with temozolomide (TMZ) and radiotherapy after surgery (Stupp protocol) increased median overall survival time to 14.6 months (3). Optune ${ }^{\circledR}$ therapy (Novocure Inc., Haifa, Israel) is a wearable technology that delivers low intensity alternating electrical fields over the scalp. Tumor-treating fields along with maximal surgical resection and Stupp regimen increases median overall survival time to 20.9 months, although it has not been used clinically in a widespread manner (4). It is important to note that median overall survival for all cases of glioblastoma is around 8 months (2).

Given the poor prognosis for patients with newly diagnosed glioblastoma, it is imperative that efforts for drug development continue in order to improve upon current standard-of-care therapies. Curcumin, a yellow pigment, derives from the rhizome of the ubiquitous and commercially available spice, turmeric (Curcuma longa). Turmeric has been long used in traditional Indian Ayurvedic medicine, with its medicinal use dating back to 2500 years ago, and more recently for its anti-inflammatory properties (5). Curcumin has also been studied for its apparent anti-tumor effects systemically. More recently, Curcumin has become an interesting consideration for glioblastoma treatment because of its modulation of multiple targets which appear to suppress tumors, decrease malignant characteristics, promote apoptosis, and potentiate the effects of chemotherapy and radiation (6-9). Interestingly, the number of peer-reviewed publications related to curcumin has been steadily increasing over the past 20 years, with an expanding portfolio of scientific reports pertaining to curcumin and gliomas. 


\section{CHEMICAL AND PHYSICAL PROPERTIES OF CURCUMIN}

Curcumin ((1E,6E)-1,7-bis(4-hydroxy-3-methoxyphenyl)-1,6-heptadiene-3,5dione) is one of three phytoconstituents (non-nutrient bioactive compound) of turmeric; the other two phytoconstituents are bis(demethoxy)curcumin and demethoxycurcumin (Figure 1). Together, these compounds account for under $10 \%$ of turmeric's dry weight $(10,11)$. After removing the protein, carbohydrates, fats, minerals, volatile oils, and fiber from raw turmeric, a crude curcuminoid extract is generated, consisting of 60-70\% curcumin, 20-27\% demethoxycurcumin, and $10-15 \%$ bis(demethoxy)curcumin.

The molecular weight of curcumin is 368.38 daltons. Curcumin's $\beta$-ketone moiety exhibits keto-enol tautomerization, with the enol form predominating in alkaline solution. It is essentially insoluble in water at room temperature and has a neutral $\mathrm{pH}$. Curcumin is photoactive and absorbs light wavelengths in the visible range of $408-500 \mathrm{~nm}$ and exhibits photodegradation. At $37^{\circ} \mathrm{C}$ the half-life at $\mathrm{pH} 7.2$ is less than 10 minutes; in vitro degradation of curcumin occurs via nucleophilic substitution or elimination by solvent molecules (solvolysis), and oxidative degradation (10).

In vivo studies indicate that the bioavailability of unaltered curcumin preparations (standard curcumin) is very poor; $12 \mathrm{~g}$ oral doses of curcumin administered to healthy human subjects produced serum concentrations of $50.5 \mathrm{ng} / \mathrm{ml}$, although other studies saw increased concentrations with concomitant administration of piperine-the alkaloid responsible for the pungency of black pepper (Piper nigrum)-indicating that bioavailability concerns can be at least partially addressed $(12,13)$. For orally ingested doses, the bioavailability issue seems to start with intestinal mucosa via modification by glucuronidation and sulfonation. Orally administered curcumin doses of $3.6 \mathrm{~g}$ are detected in colorectal tissue; however, in other studies utilizing this dose range, the serum concentration was nearly undetectable $(12,14)$. During phase I metabolism, oxidoreductases contribute to the reductive metabolism of curcumin. In phase II metabolism, intestinal epithelial cells' glucuronosyltransferases and sulfotransferases act by glucuronidation and sulfonation of curcumin. The majority of curcumin that reaches systemic circulation is reduced, conjugated, and excreted in feces, not exhibiting pharmacodynamic effects outside of the alimentary system (15).
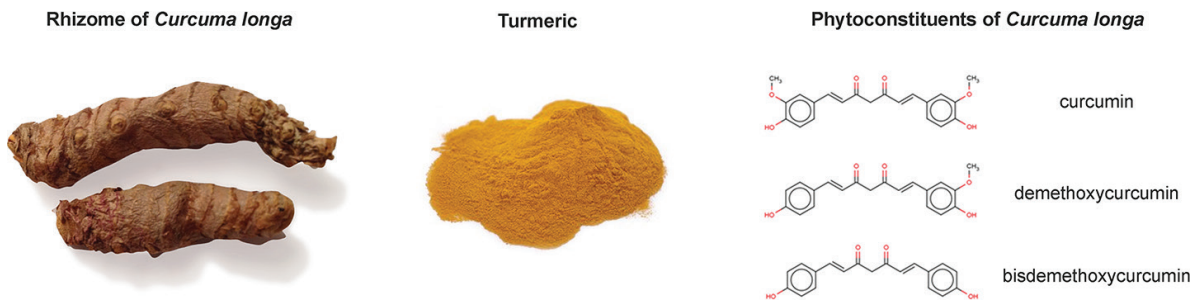

Figure 1. Curcuminoids extracted from the rhizome of turmeric (Curcuma longa) and molecular structures of the three phytoconstituents: curcumin, demethoxycurcumin, and bisdemethoxycurcumin. 


\section{TOXICITY}

Curcumin is a very well tolerated substance. Daily intake of curcumin can approach $3 \mathrm{mg} / \mathrm{kg} / \mathrm{day}$; side effects in people ingesting 500-12,000 mg doses included headache, rash, and yellow stool (16). Multiple studies regarding the safety of orally ingested curcumin have been conducted. A phase 1 trial with 25 participants ingesting $8,000 \mathrm{mg}$ curcumin per day for three months demonstrated no toxicity; five other studies using smaller doses (1,125-2,500 mg) also demonstrated curcumin's safety (17). If toxicity does occur, it is likely via curcumin's extensive interaction of hERG (human Ether-à-go-go-related gene) channels, cytochrome P450, or drug-drug interactions. In rat models, inhibition of cytochrome P450 has caused cardiotoxicity, while interaction with glutathione S-transferase has caused drug-drug interactions (10). Curcumin has been shown to induce cytotoxicity in human lymphocytes and renal cell lines at IC50 concentrations of $15.2 \mu \mathrm{M}$ and $31 \mu \mathrm{M}$, respectively (10). Currently, the FDA has not approved curcumin as a treatment for any condition and it remains classified as a safe food additive at levels from 0.5 to $100 \mathrm{mg}$ per $100 \mathrm{~g}$ of food.

\section{CURCUMIN AND SIGNAL CASCADE PROTEINS}

Curcumin appears to modulate many cellular processes, allowing it to act as an in vitro tumor suppressor, decrease malignant characteristics, promote apoptosis, and potentiate the effects of chemotherapy and radiation. Curcumin has been shown to interact with Wnt, HDGF, STAT3, and NRF2, which will be discussed in detail below.

\section{Curcumin and BIRC5}

Baculoviral inhibitor of apoptosis (BIRC5), or survivin, is a protein expressed in embryonal tissues. As a member of the inhibitor of apoptosis (IAP) family of proteins, BIRC5 is a G2/M cell cycle regulator, minimally expressed in mature tissue $(18,19)$. BIRC5 overexpression has been implicated in protection from apoptosis and regulation of mitosis, and has been associated with worse outcome in renal cell carcinoma, esophageal cancer, and breast cancer (19). In a recent study, whole genome sequencing was performed in 144 patients diagnosed with glioblastoma; those found to have high BIRC5 expression had a worse prognosis than patients with low BIRC5 expression (20). One large retrospective analysis of 1,260 patients with gliomas suggested that BIRC5 expression was correlated with worse overall survival (19). Commercially available U87, U51, and U235 human glioblastoma cell lines along with additional patient-derived glioblastoma cells were treated with $25 \mu \mathrm{M}$ curcumin solution. BIRC5 and IAP2 expression was found to be decreased after curcumin exposure at 1 and 6 hours. This effect is secondary to increased phosphorylated ERK, p38 and JNK; phosphorylated ERK inhibits STAT3, rendering it unable to translocate into the nucleus, therefore decreasing expression of BIRC5 and IAP2 (7). 


\section{Curcumin and RANK}

Receptor activator of NF-kB (RANK), via its interaction with RANKL, activates survival signaling cascades though AKT and EGFR. Alternatively, RANK can promote apoptosis via c-jun N-terminal kinase activation, also known as tumor necrosis factor receptor superfamily 11A (21). U87 and U251 glioblastoma cells exposed to $15 \mu \mathrm{M}$ and $30 \mu \mathrm{M}$ curcumin solution had a respective 1.5-2.6-fold and 1.7-3.7-fold increase in RANK mRNA levels, accomplished through inhibition of STAT3. Separately, siRNA-specific knockdown of STAT3 also produced increased RANK expression (21).

\section{Curcumin and Wnt}

The Wnt signaling pathway is a critical regulator of brain development. Presynaptic and postsynaptic transcription is regulated by Wnt; its dysfunction has been linked with development of glioblastoma (22). The Wnt signal cascade begins on the surface of cells after the bunding of the frizzled/low density lipoprotein receptor-related protein complex. A complex cascade involving Dishevelled, GSK-3 $\beta$, APC, and Axin ensues, with a subsequent intranuclear increase in $\beta$-catenin (23). The ultimate downstream effect of Wnt signaling is cell fate specification, differentiation, and mitogenic stimulation; aberrant Wnt signaling has been associated with development of glioblastoma (23). The Wnt/ $\beta$-catenin pathway contributes to cell proliferation and tumorigenesis. U373 glioblastoma cells exposed to nano-micelle curcumin preparations had suppressed Wnt, as well as NF-kB activity with an overall effect of inhibited cell growth, tumor shrinkage, and decreased invasiveness (24).

Hepatoma-derived growth factor (HDGF) is a growth factor that interacts with $\beta$-catenin to promote tumor generation, progression, and metastasis; HDGF is also upregulated in gliomas (25). The HDGF/ $\beta$-catenin complex was indirectly inhibited via inhibition of HDGF in one series with U251 and LN229 glioblastoma cells that were exposed to curcumin concentrations from 5 to $200 \mu \mathrm{mol} / \mathrm{L}$, once again resulting in reduced proliferation, invasion and extent of tumor cell migration (25).

\section{Curcumin and NRF2}

Curcumin has been reported to exhibit a radioprotective effect in normal tissues, while sensitizing tumor cells to the effects of ionizing radiation (26). One possible mechanism for this phenomenon is curcumin's interaction with nuclear factor erythroid 2 related factor 2 (NRF2). NRF2 is a leucine zipper protein that is responsible for regulation of oxidative stress; it is normally bound to KEAP1, in an inactive state. Upon exposure to insults such as toxins or radiation, NRF2 dissociates from KEAPl and accumulates within the cell's nucleus, ultimately resulting in antioxidant gene activation and resistance to oxidative stress (27). This is an important effect and suggests that curcumin may play a protective role in normal tissues that also receive systemic treatment or ionizing radiation. Curcumin is a known activator of NRF2 (27). Interestingly, NRF2 is upregulated in glioblastoma and is possibly responsible for glioblastoma survival in an environment under 
increased oxidative stress (27). Not surprisingly, U87MG glioblastoma cells treated with NRF2 had decreased levels of oxidative stress proteins, decreased proliferative capacity, and decreased self-renewal compared to controls without NRF2 knockdown.

\section{Curcumin and Protein Ubiquitination}

Protein ubiquitination is an essential process involved in regulation of many signaling cascades; ubiquitination of NEDD4 results in protein degradation (8). NEDD4 has been implicated in the development of cancers and neurodegenerative diseases and exerts an oncogenic effect via dysfunctional ubiquitin signaling (28). E3 ubiquitin ligase is a key element of the ubiquitin pathway; its aberrant function has been shown to be involved in number of malignancies (29). Neural precursor cell expressed developmentally downregulated protein 4 (NEDD4) is an important member of the E3 ubiquitin ligase family that functions in substrate recognition for the ubiquitin pathway. Activation interaction with PTEN degradation via the ubiquitin cascade eventually activates the PI3K/AKT pathway, resulting in cellular proliferation and oncogenesis (30). SNB190 and A1027 human glioblastoma cells treated with $15 \mu \mathrm{M}$ curcumin solution for 72 hours had diminished expression of NEDD4 on western blot and qPCR analysis, with reduced overall proliferative capacity (8).

S-phase kinase protein 2 (Skp2) is a component of the SKP-Cullin-F box complex, which facilitates the ubiquitin-mediated degradation of G1 checkpoint inhibitors G21, P21 and P27; the net effect is cellular proliferation via cell cycle progression $(31,32)$. Taking this into consideration, Skp2 overexpression results in unchecked cell cycle progression and has been associated with tumorigenesis, including gliomas (33-35). U251 and SNB19 glioblastoma cells treated with variable curcumin solutions demonstrated decreased Skp2 expression on RT-PCR. In line with the expected effect of decreased Skp2 expression, this study demonstrated concomitant decreases in migration, invasion, and proliferation (36). Part of curcumin's in vitro effects likely occur through modulation of protein ubiquitination, an incredibly complex and highly regulated process.

\section{CURCUMIN DECREASES TUMOR INVASIVENESS}

The malignant nature of gliomas is often due to the fact that tumor cells infiltrate deep into the parenchyma, distal to observable tumor margins; this makes complete surgical resection impossible (37). Biopsy studies in glioblastoma patients have shown that tumor cells can be found well beyond the MRI T2 hyperintense margins (38). Glioma invasion is promoted by the expression of matrix metalloproteinases (MMPs). MMPs are zinc-dependent proteolytic endopeptidases that degrade extracellular matrix proteins and cell adhesion molecules, allowing tumor cells to become locally invasive (39). Through a complex interaction with vascular endothelial growth factor (VEGF) and its receptor, VEGFR2, MMP-2 has been implicated in vasculogenesis, angiogenesis and disseminated tumor growth $(39,40)$. MMP-9 may promote vasculogenesis in the absence of VEGF $(40)$. Large diameter gliomas appear to have greater expression of MMP-2 than their smaller 
counterparts, with an accompanying decrease in expression of their inhibitor proteins (40). In one study, U373 glioblastoma cells treated with curcumin solution had measurable decreases in multiple MMPs including MMP-2 (41). Another study specifically examining invasion distance of SNB19 and A1027 glioblastoma cells after treatment with $10 \mu \mathrm{M}, 15 \mu \mathrm{M}$ and $20 \mu \mathrm{M}$ curcumin solutions found that treated cell lines had significantly less migration (invasion) than controls (8). Increased expression of MMPs may very well contribute to the invasive properties of high-grade gliomas and curcumin may be an interesting drug to combat this characteristic.

\section{CURCUMIN INDUCES OXIDATIVE STRESS AND APOPTOSIS IN GLIOMA CELL LINES}

Curcumin has been shown to have cytotoxic effects in cancer cells via the production of reactive oxygen species (ROS). U87, U51, and U235 glioblastoma lines treated with $70 \mu \mathrm{M}$ curcumin solution saw decreases in cellular viability to levels as low as $20 \%$. At curcumin concentrations as low as $25 \mu \mathrm{M}$, immunofluorescence oxidative stress assays demonstrated induction of ROS at 1 and 6 hours, normalizing after 1 day (7). U373 glioblastoma cells treated with curcumin solution demonstrated preferential induction of apoptosis at concentrations of $10 \mu \mathrm{g} / \mathrm{ml}$ and similar levels of apoptosis and necrosis and curcumin concentrations of $20 \mu \mathrm{g} / \mathrm{ml}$ (41). This is contrary to the protective effect that curcumin exhibits against oxidative stress in normal tissue (26). This dual mode of action exerted by curcumin may be due to its ability to reduce oxidative stress and inflammatory response in normal cells while also having the ability to upregulate genes responsible for cell death in pathological conditions.

\section{BARRIERS TO CURCUMIN AS A SYSTEMIC THERAPY}

Despite curcumin's apparent ability to reduce the malignant nature of glioblastoma in vitro via multiple mechanisms, it has failed to gain traction as a therapeutic agent. This, in part, may be due to curcumin's poor systemic bioavailability. In vivo bioavailability studies indicate that the bioavailability of unaltered curcumin preparations (standard curcumin) is dismal. After all, a therapeutic agent is not useful if it cannot be effectively delivered to its target in sufficient quantities. There is evidence to suggest that curcumin crosses the blood-brain barrier and may offer some neuroprotective effects, especially in context of neurodegenerative diseases such as Alzheimer's disease. In a murine model, curcumin was shown to decrease beta-amyloid plaque burden by $43 \%$ via blockage of beta amyloid self-assembly $(42,43)$. Similarly, curcumin's anti-inflammatory properties, anti-oxidant properties, metal chelation properties, and ability to decrease lipid peroxidation and lipofuscin accumulation are thought to be neuroprotective (42). The extent or mechanism of transport of curcumin across the blood-brain barrier has not been well described in humans. Common explanations in favor of curcumin's transport across the blood-brain barrier reference its lipophilic nature. However, critics are 
quick to mention that other large lipophilic molecules, such as cholesterol, cannot readily cross the blood-brain barrier. Specific transport mechanisms have not been described to date.

One of the main areas of focus for drug development of curcumin over the past years has been on preparations that increase its bioavailability. Factors that contribute to curcumin's low systemic bioavailability include its large crystalline structure, low solubility in non-toxic solution, and extensive in vivo metabolism $(15,44)$. Low-crystallinity nanoparticles were formed by exposing curcumin preparations to solvents such as chloroform. Although these preparations increased bioavailability of curcumin, their toxic solvents limited use in humans. The advent of precipitation by pressure reduction of gas-expanded liquid (PPREGL) has enabled the production of spherical curcumin nanoparticles as small as $66 \mathrm{~nm}$ (44). PPREGL nanocurcumin preparations utilize carbon dioxide instead of other volatile solvents to dry and precipitate curcumin nanoparticles from a curcumin impregnated nanoporous starch aerogel (NSA). The bioavailability of curcumin NSA nanoparticles are 173 times greater than that of standard curcumin preparations $(0.4 \%$ vs $69.1 \%)$ (44).

Other methods of curcumin preparation that increase bioavailability include liposomal curcumin and curcumin micelles. Curcumin micelles have 19-fold greater bioavailability compared to standard curcumin preparations (45). Unfortunately, micelle preparations have the drawback of a relatively large volume of stabilizers and excipients that may cause side effects such as hemolysis (46). Liposomal curcumin preparations are a promising established method of increasing curcumin bioavailability, with the ability to affix transport molecules to enhance transport across the blood-brain barrier. The p-Aminophenyl- $\alpha$-Dmannopyranoside transport molecule enhances liposomal transport across the blood-brain barrier and selectively targets cortex, hippocampus, cerebellum, brainstem, and pontine nuclei (47).

\section{CONCLUSION}

There is a dire need for advancement in glioblastoma therapy. Standard-of-care chemotherapy with temozolomide and radiation has not changed for over a decade and the prognosis for glioblastoma is dismal. Curcumin has a positive safety profile and its interaction with cell cycle regulators and regulators of oxidative stress make it an attractive possible adjunct to current standard chemotherapeutic agents and radiation in the treatment of glioblastoma. In vitro studies have demonstrated that curcumin potentiates the effects of chemotherapy and radiation on glioblastoma cells, while decreasing the ability of glioblastoma to proliferate, migrate, and recover from oxidative stress. Curcumin's main barrier to its potential use as a systemic agent is its poor bioavailability, limited by extensive modification by enterocytes and rapid metabolism. Recent progress has been made in enhancing curcumin's bioavailability by production of non-toxic nanopreparations; however, they have yet to make their way into large studies or clinical trials. While there is limited in vivo data, in vitro data suggests that curcumin can be a safe and effective anti-glioblastoma agent. Additional high-quality studies regarding the safety and efficacy of high bioavailability curcumin 
preparations will be necessary to further elucidate the potential role of curcumin in glioblastoma treatment.

Conflict of Interest: The authors declare no potential conflicts of interest with respect to research, authorship and/or publication of this article.

Copyright and Permission Statement: The authors confirm that the materials included in this chapter do not violate copyright laws. Where relevant, appropriate permissions have been obtained from the original copyright holder(s), and all original sources have been appropriately acknowledged or referenced.

\section{REFERENCES}

1. Wesseling P, Capper D. WHO 2016 Classification of gliomas. Neuropathol Appl Neurobiol. 2018;44(2):139-50. https://doi.org/10.1111/nan.12432

2. Ostrom QT, Patil N, Cioffi G, Waite K, Kruchko C, Barnholtz-Sloan JS. CBTRUS Statistical Report: Primary Brain and Other Central Nervous System Tumors Diagnosed in the United States in 20132017. Neuro-Oncology. 2020;22(Supplement_1):ivl-iv96. https://doi.org/10.1093/neuonc/noaa200

3. Stupp R, Mason WP, van den Bent MJ, Weller M, Fisher B, Taphoorn MJ, et al. Radiotherapy plus concomitant and adjuvant temozolomide for glioblastoma. N Engl J Med. 2005;352(10):987-96. https:// doi.org/10.1056/NEJMoa043330

4. Stupp R, Taillibert S, Kanner A, Read W, Steinberg DM, Lhermitte B, et al. Effect of Tumor-Treating Fields Plus Maintenance Temozolomide vs Maintenance Temozolomide Alone on Survival in Patients With Glioblastoma: A Randomized Clinical Trial. JAMA. 2017;318(23):2306-16. https://doi. org/10.1001/jama.2017.18718

5. Kocaadam B, Şanlier N. Curcumin, an active component of turmeric (Curcuma longa), and its effects on health. Crit Rev Food Sci Nutr. 2017;57(13):2889-95. https://doi.org/10.1080/10408398.2015 .1077195

6. Seyithanoğlu MH, Abdallah A, Kitiş S, Güler EM, Koçyiğit A, Dündar TT, et al. Investigation of cytotoxic, genotoxic, and apoptotic effects of curcumin on glioma cells. Cell Mol Biol (Noisy-le-grand). 2019;65(3):101-8. https://doi.org/10.14715/cmb/2019.65.3.15

7. Gersey ZC, Rodriguez GA, Barbarite E, Sanchez A, Walters WM, Ohaeto KC, et al. Curcumin decreases malignant characteristics of glioblastoma stem cells via induction of reactive oxygen species. BMC Cancer. 2017;17(1):99. https://doi.org/10.1186/s12885-017-3058-2

8. Wang X, Deng J, Yuan J, Tang X, Wang Y, Chen H, et al. Curcumin exerts its tumor suppressive function via inhibition of NEDD4 oncoprotein in glioma cancer cells. Int J Oncol. 2017;51(2):467-77. https://doi.org/10.3892/ijo.2017.4037

9. Fratantonio D, Molonia MS, Bashllari R, Muscarà C, Ferlazzo G, Costa G, et al. Curcumin potentiates the antitumor activity of Paclitaxel in rat glioma C6 cells. Phytomedicine. 2019;55:23-30. https://doi. org/10.1016/j.phymed.2018.08.009

10. Nelson KM, Dahlin JL, Bisson J, Graham J, Pauli GF, Walters MA. The Essential Medicinal Chemistry of Curcumin. J Med Chem. 2017;60(5):1620-37. https://doi.org/10.1021/acs.jmedchem.6b00975

11. Horosanskaia E, Yuan L, Seidel-Morgenstern A, Lorenz H. Purification of Curcumin from Ternary Extract-Similar Mixtures of Curcuminoids in a Single Crystallization Step. Crystals. 2020;10:206. https://doi.org/10.3390/cryst10030206

12. Anand P, Kunnumakkara AB, Newman RA, Aggarwal BB. Bioavailability of Curcumin: Problems and Promises. Molecular Pharmaceutics. 2007;4(6):807-18. https://doi.org/10.1021/mp700113r

13. Shoba G, Joy D, Joseph T, Majeed M, Rajendran R, Srinivas PS. Influence of piperine on the pharmacokinetics of curcumin in animals and human volunteers. Planta Med. 1998;64(4):353-6. https://doi. org/10.1055/s-2006-957450 
14. Sharma RA, Steward WP, Gescher AJ. Pharmacokinetics and pharmacodynamics of curcumin. Adv Exp Med Biol. 2007;595:453-70. https://doi.org/10.1007/978-0-387-46401-5_20

15. Heger M, van Golen RF, Broekgaarden M, Michel MC. The Molecular Basis for the Pharmacokinetics and Pharmacodynamics of Curcumin and Its Metabolites in Relation to Cancer. Pharmacological Reviews. 2014;66(1):222. https://doi.org/10.1124/pr.110.004044

16. Hewlings SJ, Kalman DS. Curcumin: A Review of Its' Effects on Human Health. Foods. 2017;6(10):92. https://doi.org/10.3390/foods6100092

17. Chainani-Wu N. Safety and anti-inflammatory activity of curcumin: a component of tumeric (Curcuma longa). J Altern Complement Med. 2003;9(1):161-8. https://doi.org/10.1089/107555303321223035

18. Garg H, Suri P, Gupta JC, Talwar GP, Dubey S. Survivin: a unique target for tumor therapy. Cancer Cell International. 2016;16(1):49. https://doi.org/10.1186/s12935-016-0326-1

19. Zhang S, Zhang C, Song Y, Zhang J, Xu J. Prognostic role of survivin in patients with glioma. Medicine (Baltimore). 2018;97(17):e0571. https://doi.org/10.1097/MD.0000000000010571

20. Tong X, Yang P, Wang K, Liu Y, Liu X, Shan X, et al. Survivin is a prognostic indicator in glioblastoma and may be a target of microRNA-218. Oncol Lett. 2019;18(1):359-67. https://doi.org/10.3892/ ol.2019.10335

21. Wu B, Yao X, Nie X, Xu R. Epigenetic reactivation of RANK in glioblastoma cells by curcumin: involvement of STAT3 inhibition. DNA Cell Biol. 2013;32(6):292-7. https://doi.org/10.1089/dna.2013.2042

22. Zuccarini M, Giuliani P, Ziberi S, Carluccio M, Iorio PD, Caciagli F, et al. The Role of Wnt Signal in Glioblastoma Development and Progression: A Possible New Pharmacological Target for the Therapy of This Tumor. Genes (Basel). 2018;9(2):105. https://doi.org/10.3390/genes9020105

23. Logan CY, Nusse R. The Wnt signaling pathway in development and disease. Annu Rev Cell Dev Biol. 2004;20:781-810. https://doi.org/10.1146/annurev.cellbio.20.010403.113126

24. Hesari A, Rezaei M, Rezaei M, Dashtiahangar M, Fathi M, Rad JG, et al. Effect of curcumin on glioblastoma cells. J Cell Physiol. 2019;234(7):10281-8. https://doi.org/10.1002/jcp.27933

25. Luo Q, Luo H, Fu H, Huang H, Huang H, Luo K, et al. Curcumin suppresses invasiveness and migration of human glioma cells in vitro by inhibiting HDGF/ $\beta$-catenin complex. Nan fang yi ke da xue xue bao = Journal of Southern Medical University. 2019;39(8):911-6.

26. Jagetia GC. Radioprotection and radiosensitization by curcumin. Adv Exp Med Biol. 2007;595: 301-20. https://doi.org/10.1007/978-0-387-46401-5_13

27. Godoy PRDV, Pour Khavari A, Rizzo M, Sakamoto-Hojo ET, Haghdoost S. Targeting NRF2, Regulator of Antioxidant System, to Sensitize Glioblastoma Neurosphere Cells to Radiation-Induced Oxidative Stress. Oxidative medicine and cellular longevity. 2020;2020:2534643. https://doi. org/10.1155/2020/2534643

28. Faktor J, Pjechová M, Hernychová L, Vojtěšek B. Protein Ubiquitination Research in Oncology. Klin Onkol. 2019;32(Supplementum 3):56-64. https://doi.org/10.14735/amko20193S56

29. Zou X, Levy-Cohen G, Blank M. Molecular functions of NEDD4 E3 ubiquitin ligases in cancer. Biochim Biophys Acta. 2015;1856(1):91-106. https://doi.org/10.1016/j.bbcan.2015.06.005

30. Wang X, Trotman LC, Koppie T, Alimonti A, Chen Z, Gao Z, et al. NEDD4-1 is a proto-oncogenic ubiquitin ligase for PTEN. Cell. 2007;128(1):129-39. https://doi.org/10.1016/j.cell.2006.11.039

31. Deng T, Yan G, Song X, Xie L, Zhou Y, Li J, et al. Deubiquitylation and stabilization of p21 by USP11 is critical for cell-cycle progression and DNA damage responses. Proc Natl Acad Sci U S A. 2018;115(18):4678-83. https://doi.org/10.1073/pnas.1714938115

32. Wu J, Su HK, Yu ZH, Xi SY, Guo CC, Hu ZY, et al. Skp2 modulates proliferation, senescence and tumorigenesis of glioma. Cancer Cell Int. 2020;20:71. https://doi.org/10.1186/s12935-020-1144-z

33. Cai Z, Moten A, Peng D, Hsu CC, Pan BS, Manne R, et al. The Skp2 Pathway: A Critical Target for Cancer Therapy. Semin Cancer Biol. 2020. https://doi.org/10.1016/j.semcancer.2020.01.013

34. Gstaiger M, Jordan R, Lim M, Catzavelos C, Mestan J, Slingerland J, et al. Skp2 is oncogenic and overexpressed in human cancers. Proc Natl Acad Sci U S A. 2001;98(9):5043-8. https://doi.org/10.1073/ pnas.081474898

35. Zhang W, Cao L, Sun Z, Xu J, Tang L, Chen W, et al. Skp2 is over-expressed in breast cancer and promotes breast cancer cell proliferation. Cell Cycle. 2016;15(10):1344-51. https://doi.org/10.1080/ 15384101.2016.1160986 
36. Wang L, Ye X, Cai X, Su J, Ma R, Yin X, et al. Curcumin suppresses cell growth and invasion and induces apoptosis by down-regulation of Skp2 pathway in glioma cells. Oncotarget. 2015;6(20):18027-37. https://doi.org/10.18632/oncotarget.4090

37. Smith SJ, Diksin M, Chhaya S, Sairam S, Estevez-Cebrero MA, Rahman R. The Invasive Region of Glioblastoma Defined by 5ALA Guided Surgery Has an Altered Cancer Stem Cell Marker Profile Compared to Central Tumour. Int J Mol Sci. 2017;18(11):2452. https://doi.org/10.3390/ ijms 18112452

38. Price SJ, Gillard JH. Imaging biomarkers of brain tumour margin and tumour invasion. Br J Radiol. 2011;84 Spec No 2(Spec Iss 2):S159-S67. https://doi.org/10.1259/bjr/26838774

39. Guo G, Sun Y, Hong R, Xiong J, Lu Y, Liu Y, et al. IKBKE enhances TMZ-chemoresistance through upregulation of MGMT expression in glioblastoma. Clin Transl Oncol. 2019. https://doi.org/10.1007/ s12094-019-02251-3

40. Tabouret E, Boudouresque F, Farina P, Barrié M, Bequet C, Sanson M, et al. MMP2 and MMP9 as candidate biomarkers to monitor bevacizumab therapy in high-grade glioma. Neuro-oncology. 2015;17(8):1174-6. https://doi.org/10.1093/neuonc/nov094

41. Abdullah Thani NA, Sallis B, Nuttall R, Schubert FR, Ahsan M, Davies D, et al. Induction of apoptosis and reduction of MMP gene expression in the U373 cell line by polyphenolics in Aronia melanocarpa and by curcumin. Oncol Rep. 2012;28(4):1435-42. https://doi.org/10.3892/or.2012.1941

42. Mishra S, Palanivelu K. The effect of curcumin (turmeric) on Alzheimer's disease: An overview. Ann Indian Acad Neurol. 2008;11(1):13-9. https://doi.org/10.4103/0972-2327.40220

43. Yang F, Lim GP, Begum AN, Ubeda OJ, Simmons MR, Ambegaokar SS, et al. Curcumin inhibits formation of amyloid beta oligomers and fibrils, binds plaques, and reduces amyloid in vivo. J Biol Chem. 2005;280(7):5892-901. https://doi.org/10.1074/jbc.M404751200

44. Ubeyitogullari A, Ciftci ON. A novel and green nanoparticle formation approach to forming lowcrystallinity curcumin nanoparticles to improve curcumin's bioaccessibility. Scientific Reports. 2019;9(1):19112. https://doi.org/10.1038/s41598-019-55619-4

45. Zhang Q, Polyakov NE, Chistyachenko YS, Khvostov MV, Frolova TS, Tolstikova TG, et al. Preparation of curcumin self-micelle solid dispersion with enhanced bioavailability and cytotoxic activity by mechanochemistry. Drug Deliv. 2018;25(1):198-209. https://doi.org/10.1080/10717544. 2017.1422298

46. Li X, Yuan H, Zhang C, Chen W, Cheng W, Chen X, et al. Preparation and in-vitro/in-vivo evaluation of curcumin nanosuspension with solubility enhancement. J Pharm Pharmacol. 2016;68(8):980-8. https://doi.org/10.1111/jphp.12575

47. Wang Y, Ying X, Xu H, Yan H, Li X, Tang H. The functional curcumin liposomes induce apoptosis in C6 glioblastoma cells and C6 glioblastoma stem cells in vitro and in animals. Int J Nanomedicine. 2017;12:1369-84. https://doi.org/10.2147/IJN.S124276 
